\title{
ANÁLISIS BURSÁTIL SECTORIAL CON RELACIÓN A LA TASA DE INTERÉS EN MÉXICO
}

\section{SECTORAL STOCK MARKET ANALYSIS IN RELATION TO THE INTEREST RATE IN MEXICO}

Mtro. Mario Aceves Mejía ${ }^{a}$

C. Ashley Daniela Torres Carrasco ${ }^{b}$

Dra. Rufina Georgina Hernández Contreras ${ }^{c}$

\author{
${ }^{a}$ Benemérita Universidad Autónoma de Puebla \\ Facultad de Contaduría Pública, acevesmm@gmail.com \\ ${ }^{\mathrm{b}}$ Universidad Autónoma de Nayarit \\ Unidad Académica de Contaduría y Administración, ashleytcarrasco@gmail.com \\ ${ }^{\mathrm{c}}$ Benemérita Universidad Autónoma de Puebla \\ Facultad de Contaduría Pública, ghernandez0727@hotmail.com
}

Fecha de recepción: 30 de abril de 2020

Fecha de aceptación: 25 de mayo de 2020

\section{RESUMEN}

El presente trabajo de investigación tiene por objetivo integrar a discusión un estudio en términos teóricos y fundamentado con base en evidencia empírica, sobre la relación entre la política monetaria de objetivos de inflación del Banco de México con respecto al mercado bursátil agregado y desagregado, reparando en el uso de una metodología cortoplacista impulso-respuesta, por medio de modelos econométricos de Vectores Autorregresivos basados en tasas de crecimiento. De esta forma, fueron considerados los mecanismos de trasmisión entre la tasa de interés (TIIE) con relación al IPC de la Bolsa Mexicana de Valores, así como la segregación sectorial de este último, de manera que fue posible pronosticar y definir estrategias de inversión factibles con base en las decisiones de política monetaria, amplificando el proceder de la dirección financiera corporativa-bursátil.

PALABRAS ClAVE: Tasa de interés; Índice de precios y cotizaciones; Política monetaria. 


\begin{abstract}
The objective of this research work is to integrate a discussion in theoretical terms and based on empirical evidence on the relationship between the monetary policy of inflation objectives of Banco de México regard to the aggregate and disaggregated stock market, ponder on the use of a short-term impulse-response methodology, by econometric models of Autoregressive Vectors based on growth rates. In this way, the transmission mechanisms between the interest rate (TIIE) in relation to the IPC of Bolsa Mexicana de Valores were considered, as well as the sectoral segregation of the latter, so that it was possible to forecast and define feasible investment strategies with based on monetary policy decisions, amplifying the actions of the corporate-stock market financial management.
\end{abstract}

KEYWORDS: Interest rate; Price index and quotations; Monetary policy.

\title{
I. INTRODUCCIÓN
}

Desde hace algunos años, la dirección financiera de las empresas ha basado sus decisiones de inversión bursátil exclusivamente en modelos de volatilidad de precios o índices, relegando la importancia de la dinámica macroeconómica, así como a los mecanismos de transmisión que definen el comportamiento de sus distintos indicadores como lo es la política monetaria y su afectación sobre la tasa de interés; la cual a su vez se relaciona negativamente con la inversión en instrumentos pertenecientes al mercado de capitales (acciones, certificados de participación, etc.).

Al respecto, diversos bancos centrales, incluido el Banco de México, han adoptado el uso de reglas monetarias fundamentadas en el Modelo de Inflación Objetivo (MIO) (Perrotini, 2014, Woodford, 2012), el cual busca reducir y mantener constante a la tasa de inflación por medio de incrementos en la tasa de interés y la restricción monetaria. No obstante, dicha dirección de política considera que la inflación es una problemática per se monetaria, ignorando que puede producir efectos acumulativos y diferenciados sobre la economía real, y por ende en la dinámica bursátil de los mercados de valores. 
De esta forma, el presente artículo persigue un análisis teórico-empírico que procura como hipótesis la relación de causalidad perjudicial entre la dinámica de la Tasa de Interés Interbancaria de Equilibrio (TIIE) determinada por Banco de México sobre el crecimiento bursátil, mediante el Índice de Precios y Cotizaciones de la Bolsa Mexicana de Valores, así como por el desglose sectorial de este último, dado que el carácter diferenciado de la tasa de interés sobre las distintas ramas productivas en México. Es así que, de inicio se considera un análisis sobre la interacción de la dinámica de tasas de interés con respecto al sector bursátil, para seguir con una evaluación de hechos estilizados correspondientes a la conducta macroeconómica y bursátil de la economía mexicana; continuando con la comprobación y recuento de las estimaciones econométricas por medio de un conjunto de modelos impulso-respuesta de Vectores Autorregresivos (VAR, por sus siglas en inglés) y finalizando con las conclusiones derivadas de la presente investigación, presentando propuestas de inversión benéficas y factibles en estos tiempos de incertidumbre ante el análisis de política monetaria de Banxico, y así considerar sectores del IPC sensibles e insensibles a la misma.

\section{ANÁLISIS REFERENCIAL, ESTUDIOS MACROECONÓMICOS- BURSÁTILES}

Tradicionalmente la teoría general de finanzas y desarrollo establece una estrecha relación entre el crecimiento financiero y el desarrollo económico. A saber, dicho corpus teórico postula una relación directa entre el mercado bursátil y el desempeño económico de la nación, de la que resulta un impacto bidireccional entre los sectores involucrados (Gómez, 2010).

Al respecto, existe cierto debate teórico-empírico sobre la relación causal entre la dinámica de algunas variables macroeconómicas como lo son la inflación, la dinámica de las tasas de interés, o el tipo de cambio con respecto al crecimiento del sector financiero bursátil (Gómez, 2010; Velázquez y Hernández , 2013). 
En particular, Velázquez y Hernández (2013) expresan que el modelo de equilibrio general, el sistema financiero influye sobre el sector real de la economía a través la vinculación de los ahorros de las familias con los requerimientos de fondos prestables para financiar la inversión que realizan las empresas. De esta manera, los ahorros de las familias se convierten en créditos para las empresas que requieren financiación; de esta forma, la dinámica bursátil depende del proceder de política macroeconómica.

No obstante, Pagano (1993) y Levine (2004) extienden al estudio de crecimiento endógeno y considera los lineamientos del principio de causalidad, resaltando la funcionalidad de analizar en profundidad cómo es que impactan las funciones del sistema financiero sobre la dinámica macroeconómica y viceversa, donde particularmente la dinámica de los mercados accionarios tiene una afectación reducida sobre la economía real, mientras que la economía real produce afectaciones significativas sobre el comportamiento de los mercados financieros.

En cuanto a estudios de pruebas econométricas, Levine (2004) implementa indicadores financieros como la ratio del dinero y el PIB, identificando la causalidad bidireccional, especialmente en países industrializados. A su vez, también se plantea un modelo no lineal que refleja una curva de desigualdad entre el ingreso y desarrollo de los intermediarios financieros bursátiles y donde particularmente el sector de mayor valor agregado, que es la industria, es quien impulsa la dinámica de los mercados accionarios, mientras que el movimiento contrario es particularmente insignificante; y por lo tanto, se tiene un problema especulativo en los países desarrollados donde el sector financiero ha perdido sus cualidades de impulsar a la economía real y ésta última en realidad es quien amplifica el margen de capitalización especulativa.

En otro aspecto, en cuanto a los mecanismos de transmisión del Modelo de Inflación Objetivo y su fundamental más significativo que es la Regla de Taylor, este paradigma macroeconómico analiza la relación entre la tasa de interés y la inflación meta del Banco Central, así como otras variables de los agregados económicos que estructuran las políticas 
monetarias. A saber, dicha regla fue desarrollada en Estados Unidos de América en la década de 1990 y ha sido aplicada por la Reserva Federal, a fin de lograr la estabilidad del crecimiento de los precios, reduciendo los niveles de especulación y a su vez reducir la vulnerabilidad de políticas macroeconómicas discrecionales (Perrotini, 2014; Sánchez, 2020; Taylor 1993, 1998; Woodford, 2012). En lo particular, Taylor $(1993,1998)$ consideró un consenso de distintos enfoques macroeconómicos que se sintetizan de la siguiente forma:

$$
\mathrm{R}_{\mathrm{t}}=\tilde{\mathrm{r}}+\mathrm{a}\left(\pi_{\mathrm{t}}-\bar{\pi}\right)+\beta\left(\mathrm{Y}_{\mathrm{t}}-\overline{\mathrm{Y}}\right)
$$

Donde:

$R_{t}=$ es la tasa de interés real.

$\left(\pi_{t}-\bar{\pi}\right)=$ es la diferencia de los precios observados y la meta de inflación objetivo.

$\left(Y_{t}-\bar{Y}\right)=$ es la diferencia del producto observado y del producto potencial.

Dicha ecuación (1) expresa que, cuando incrementa el producto efectivo por encima de su nivel potencial, la inflación tiende a exceder su meta y por lo tanto la tasa de interés deberá elevarse para reducir la brecha de producto e inflación. A su vez, esta ecuación permite un rango de flexibilidad, logrando disminuir los valores de los parámetros para incrementar la positividad al aplicarla en el estudio de distintos Bancos Centrales.

Para el caso de la evidencia empírica del seguimiento del enfoque macroeconómico de Objetivos de Inflación en la economía mexicana se tienen diversos estudios como son los trabajos de Figueroa, Pérez y Godínez (2016) García y Perrotini (2014), Perrotini (2014) y Sánchez (2020), los cuales han demostrado que el seguimiento de la política monetaria de Banxico requiere de un uso de los incrementos de la tasa de interés por arriba de la tasa de aceleración inflacionaria con un diferencial cada vez más amplio, reflejando la pérdida de efectividad de contención del tipo de cambio, el efecto traspaso y logro del objetivo inflacionario de $3 \%$ con el paso del tiempo, es decir, cada vez más se pierde eficiencia en el uso de la regla monetaria. 
Por todo lo anterior, es posible deducir que, si la tasa de interés se utiliza para contener a la demanda agregada, y que para el caso de México ese mecanismo se utiliza con mayor escala y un número de repeticiones incrementado, la dinámica bursátil debe presentar respuestas ante estos impulsos. A saber, estudios como los de Basistha y Startz (2020) Hallberg y Ryhage (2019), consideran para países desarrollados que el seguimiento de regla monetaria del Modelo de Inflación Objetivo presenta cierto grado de sustituibilidad entre los instrumentos relacionados con la tasa de interés, con respecto a aquellos pertenecientes al mercado de capitales; sin embargo, estos efectos son bastante heterogéneos y no presentan un análisis sectorial, concluyendo exclusivamente que ante los incrementos de la tasa de interés, los mercados de valores presentan reducciones en sus índices, sin embargo, es relevante aclarar que el incumplimiento de meta de inflación en estos estudios se muestra en ocasiones muy limitadas, siendo investigaciones con un conjunto muestra mayormente estable.

Particularmente, la dinámica de la política monetaria en México ha presentado distintos cambios a lo largo de los últimos 30 años, siendo la inflación el principal objetivo del Banco de México, siguiendo el principio del Modelo de Inflación Objetivo y la Regla de Taylor.

Para conocer de forma detallada la trayectoria del seguimiento de la política monetaria en México, y así definir la relación con respecto al comportamiento bursátil, es necesario describir y analizar la dinámica de la Inflación, la Tasa de Interés Interbancaria de Equilibrio (TIIE), así como el tipo de cambio como sigue en la figura 1: 


\section{Figura 1.}

Inflación, México, 1997 a 2020.

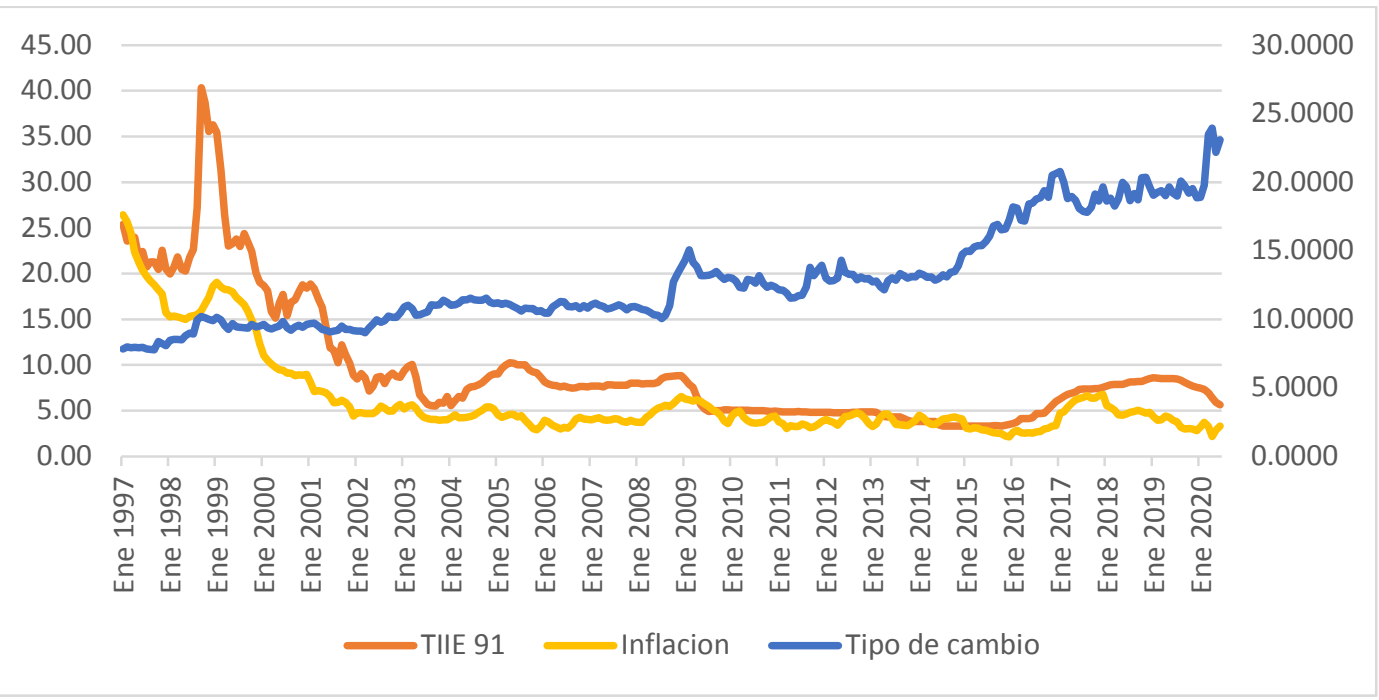

Elaboración propia con base en Banxico (2020) e INEGI (2020).

Al respecto, la gráfica 1 considera de forma conjunta a los elementos de la política monetaria en México, de la cual es posible considerar que la inflación representa una dinámica decreciente frente a la actividad de los años noventa del siglo $\mathrm{XX}$, cuando el índice inflacionario se posicionaba por encima de los 25 puntos porcentuales derivado entre muchos otros factores por los rezagos de la implementación de una nueva unidad monetaria denominada "nuevo peso", y que para lograrlo se desarrolló en 1997 un programa monetario que tenía por objeto disminuir la tasa de inflación en 15\%, lo cual facilitó el ajuste a la baja de las expectativas de inflación. Sin embargo, durante los últimos 21 años la economía mexicana no ha logrado estabilizar la brecha de producción, por lo que la inflación durante este periodo se muestra fluctuante, pero en menor escala que hace dos décadas, logrando una tasa de inflación del $2.83 \%$ en 2019, el nivel más bajo desde el año 2016, pero que ha estado fuera de su meta en un mayor número de ocasiones que dentro de la misma.

En cuanto al tipo de cambio, la estructura financiera en México ha experimentado cambios radicales como resultado de las transformaciones en el mismo y otros sistemas financieros a lo largo de la historia, y la modificación del régimen cambiario en la década de los 90’s 
también implicó un nivel de volatilidad mayor en nuestra moneda, sin embargo, el peso mexicano jamás había presentado un nivel de depreciación frente al dólar tan alto como en el año actual. Es evidente la constante tendencia creciente sobre la pérdida de valor de nuestra moneda, pasando de \$7.91 MXN por dólar en 1997 a \$19.26 MXN por dólar en 2019 (gráfica 1), lo que demuestra que, a pesar de que gráficamente la tasa de inflación y el tipo de cambio presentan trayectorias contrarias, su correlación está definida por sus tasas de crecimiento. A saber, a pesar de que tienen trayectorias contrarias.

Finalmente, la gráfica 1 considera la trayectoria de la Tasa de Interés Interbancaria de Equilibrio (TIIE), determinada por el Banco de México, mostrando una tendencia a la baja comparando las cifras de su nivel más alto en 1999 de $40 \%$ a 2019 donde se posiciona en $7.5 \%$, pasando por su nivel más bajo entre 2014 y finales de 2015 de 3.3\%. A saber, dicha variable representa en promedio las tasas de interés que los bancos cobran por sus créditos, los cuales deberán verse afectados en relación inversa a esta variable, por lo que, si la TIIE tiende a disminuir se incrementan los componentes de la demanda agregada. Sin embargo, la tasa actual del Banco de México (y desde 2016) ha sido una de las más altas en la última década, derivado de una creciente inflación que en 2017 se ubicó por encima del 4\% y una dinámica como estrategia de BANXICO donde se incrementa la TIIE a fin de mantener estable el tipo de cambio de 2016 a 2019.

Es así que, si bien la dinámica del MIO se ha llevado a cabo de manera satisfactoria, es decir, se ha logrado el objetivo de política monetaria de una inflación de 3\% en términos promedio, es observable que el uso de la Regla de Taylor es cada vez más recurrente con el incremento cada vez más amplificado de la TIIE.

Ahora bien, bajo la consideración del análisis del sector bursátil es pertinente iniciar con la trayectoria del IPC general de la Bolsa Mexicana de Valores. En términos generales, la dinámica de comportamiento del IPC se muestra creciente considerando los niveles alcanzados a finales de los años noventa y principios de la segunda década de los 2000's, cuando el índice se encontraba cerca de los 5,000 puntos a diferencia de los 36,000 puntos a 
mediados de 2020, dinámica que además se enfatiza por medio de un modelo autorregresivo de tendencia con una bondad de ajuste de 0.9078 , como se observa en la figura 2:

Figura 2.

Índice de Precios y Cotizaciones, Bolsa Mexicana de Valores, 1998-2020.

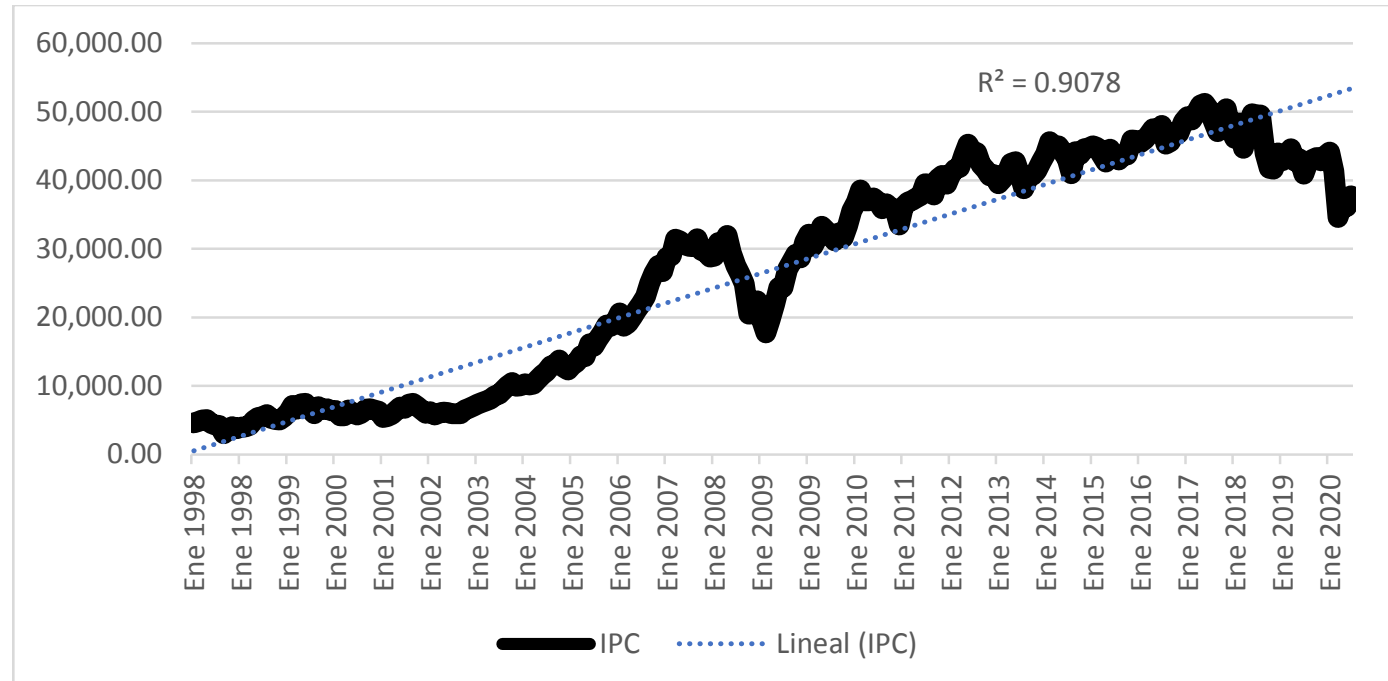

Elaboración propia con base en Bolsa Mexicana de Valores (2020).

Si bien, el IPC ha mostrado una dinámica con una tendencia creciente logrando su punto más alto en septiembre de 2017, desde febrero de 2013 se han presentado contracciones significativas, además de identificar un estancamiento y reducción que rondan a los 40,000 puntos del IPC, así desde enero de 2018 hasta junio de 2020 se logra identificar una relación de comportamiento inversa entre el Índice de Precios y Cotizaciones y la dinámica de la TIIE (vista en la figura 1). De esta forma, posterior al punto más álgido del IPC, la tasa de interés tuvo una aceleración importante pasando de 3.5\% a $8.5 \%$ de diciembre de 2015 a diciembre de 2018, periodo donde el IPC ha presentado precisamente una debacle.

Ahora bien, en cuanto a la dinámica del desglose sectorial del IPC, la Bolsa Mexicana de Valores ha determinado dicha categorización como sigue: Materiales, Industrial, Servicios y Bienes de Consumo Básico, Servicios y Bienes de Consumo Frecuente, Salud, Servicios Financieros, y Servicios de Telecomunicaciones. La figura 3 presenta que la dinámica 
sectorial ha presentado un comportamiento diferenciado, empezando con el sector de materiales este es creciente, pero en términos escalonados rondando los 600 puntos desde año 2008, y repitiendo dicho valor esperando en 2015 y 2020.

\section{Figura 3.}

Índice de Precios y Cotizaciones, Sector Materiales, Bolsa Mexicana de Valores, 1998-2020.

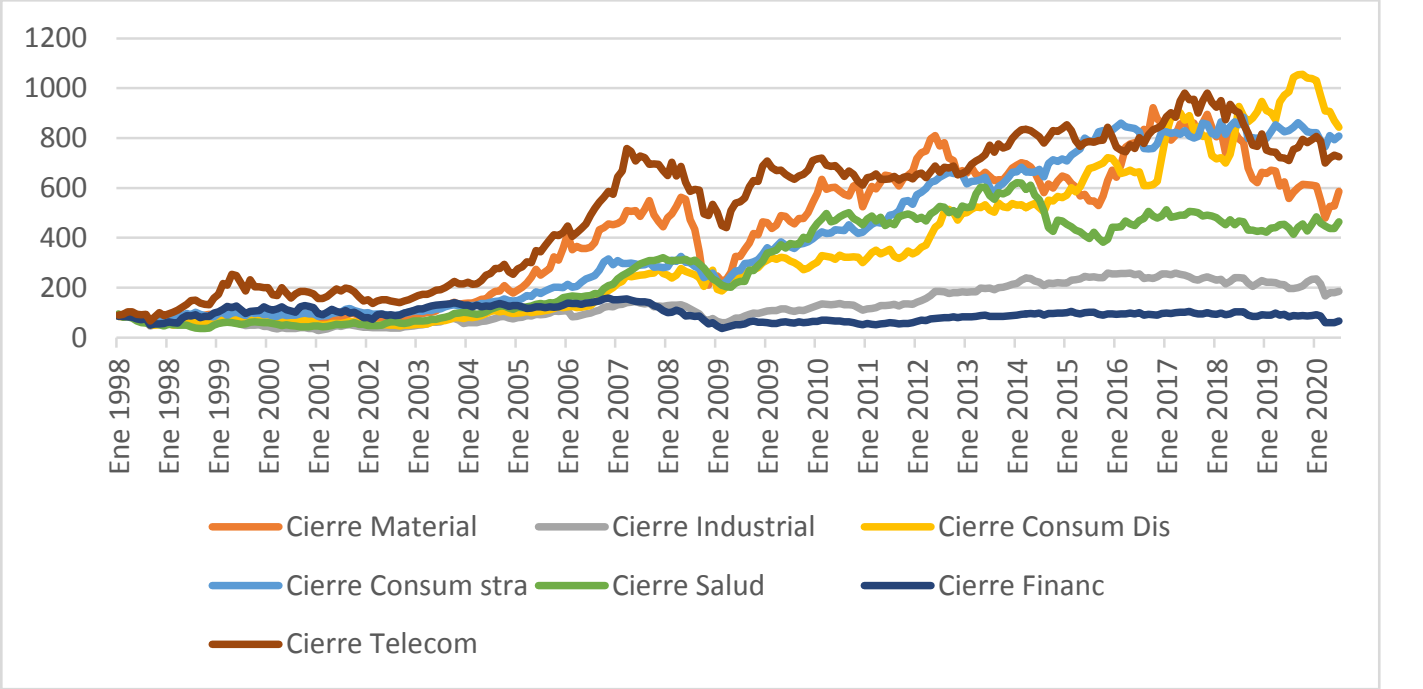

Elaboración propia con base en Bolsa Mexicana de Valores (2020).

Por su parte, el IPC del Sector Industrial muestra una tendencia moderadamente creciente al considerar la dinámica del año 1998 respecto al comportamiento actual, donde el nivel ha incrementado solamente cerca de los 100 puntos, sin olvidar los rezagos que implicó la caída de los mercados internacionales en 2008, o bien los mayores niveles alcanzados entre enero de 2015 y 2017, ubicando a este sector por encima de las 250 unidades a excepción del año 2016. En 2019 se presentó uno de los mayores decrementos en el índice desde 2013, sin embargo, el nivel actual mantiene la tendencia a la alta respecto a los valores iniciales de la gráfica.

En cuanto al Sector de Servicios y Bienes de Consumo No Básico del IPC, éste es uno de los sectores que muestra mayor estabilidad, manteniendo una trayectoria de crecimiento significativo, sin dejar de mencionar que la afectación que marcó a otros sectores en 2008 no tuvo mayor significancia para éste, como sí lo fue en entre 2017 y mediados del 2020, después de alcanzar su punto máximo en 2019, por encima de los 1000 puntos. 
Ahora bien, el IPC del Sector Servicios y Bienes de Consumo Básico muestra una dinámica de crecimiento positiva y en mayor escala, considerando un incremento en las últimas dos décadas en poco más de 700 puntos logrando para mediados del 2020 un nivel de posicionamiento de alrededor 800 puntos IPC, lo que demuestra su tendencia creciente y relación de comportamiento con la interacción del IPC general, pero en un nivel de sensibilidad menor que en otros sectores. Sin embargo, el Sector de Servicios y Bienes de Consumo Básico alcanzó sus niveles máximos de casi 900 entre los años 2016 y 2018.

En otro sentido, el Sector Salud muestra una tendencia ascendente logrando a más de 450 puntos para mediados del 2020, alcanzando el registro más elevado en décadas en el periodo comprendido entre enero de 2013 y 2014 con alrededor de 600 puntos, nivel máximo identificado justo antes de presenciar una de las mayores caídas del indicador después de haber mantenido un nivel por encima de los 400 puntos por casi 5 años consecutivos, declive del cuál no se ha recuperado hasta el momento.

Acerca del IPC del Sector de Servicios Financieros, la gráfica 3 muestra que éste tiene una tendencia decreciente si consideramos su dinámica desde 1998, cuando se posicionaba en cuantías aproximadas a los 85 puntos, hasta mediados del 2020 donde el índice esta por alcanzar un valor grafico de 70. Atravesando por un mínimo histórico en 2008 con un nivel de casi los 40 puntos y un máximo de cerca 160 puntos entre enero de 2007 y principios de 2008, un periodo antes de la crisis que afectaría a gran parte de los mercados y economías internacionales y que establecería una de las mayores caídas financieras de la historia moderna. A su vez, el presente indicador muestra en su comportamiento de las últimas dos décadas un nivel de sensibilidad al IPC general muy por debajo del resto de los sectores, así como múltiples caídas registradas en la BMV relacionadas con los crecimientos de la Tasa de Interés Interbancaria de Equilibrio.

Finalmente, para el caso del IPC Servicios de Telecomunicaciones, éste ha logrado mantenerse entre los 700 puntos desde enero de 2019 hasta la fecha. No obstante, presenta un alto grado de sensibilidad reflejado entre 2008 y 2009 al decrecer cerca de 200 puntos 
desde el año anterior (2007), lo que significaría la presencia de variaciones frente al tipo de cambio y a las tasas de interés.

\section{METODOLOGÍA, MODELOS ECONOMÉTRICOS DE VECTORES AUTORREGRESIVOS}

Como ha sido abordado con anterioridad, el desarrollo del análisis de la relación entre el crecimiento bursátil y la tasa de interés es un problema creciente que, en la actualidad, se presenta relevante sobre la discusión en temas de interés particular, constituido a partir de las interacciones dinámicas temporales entre las variables, las cuales han determinado el desarrollo causal de un sistema subyacente y las condiciones para realizar pronósticos. Comúnmente surgen complicaciones al calificar si la interacción de las variables resulta ser la causa de la variación de otras consideradas, pues constantemente en los trabajos de investigación empíricos suele omitirse por suposición esta parte y se dirige al desarrollo directo de la relación entre ellas, encontrándonos así con un gran número de aplicaciones econométricas con excesivos ajustes entre variables que reflejan relaciones carentes de verdadero significado en la economía real.

El concepto probabilístico de causalidad se fundamenta en el principio que prescribe que, en el tiempo, la causa siempre precede a los efectos. Granger (1969) fue quien tuvo la primicia de establecer criterios validos sobre dicho efecto, argumentando que el futuro no es capaz de incidir en hechos del pasado, sino que ocurre de manera inversa. De esta forma, si una variable retardada muestra correlación a valores futuros de otra variable, se dice que es causa de la otra.

Sin embargo, establecer que solo por ello existe causalidad no es idóneo, pues existe la posibilidad que una variable retardada se correlacione espuriamente con otra variable solo porque es un indicador adelantado y no precisamente porque exista verdadera causalidad, sobre todo si son series temporales no estacionarias (especulación); en cualquier caso, lo que sí es posible afirmar es que, si no existe dicha correlación, entonces la variable retardada no será la causa a la otra (Montero, 2013). 
De esta manera, la hipótesis a considerar en la presente investigación estipula que la dinámica de la política monetaria es un determinante del proceder del sistema financiero bursátil; por lo tanto, la comprobación del mecanismo de transmisión entre la dinámica bursátil con relación a la Tasa de interés para el caso de México se basaría en dos condiciones: 1) el IPC, así como su comportamiento desglosado en sectores, guarda relación directa con los objetivos inflacionarios del Banco Central; mientras que 2) la sustitución entre instrumentos financieros pertenecientes al mercado de capitales con respecto al mercado de dinero, incentiva a la sucesión de los componentes de los índices de capitalización hacía instrumentos de deuda por medio del uso de la regla de Taylor (1993, 1998).

Es así, que inicialmente la forma funcional de los modelos en cuestión es:

$$
I P C_{i, t}=b_{1}+b_{2} * T I I E_{t} \quad(\text { EC. } 2)
$$

Donde la variable independiente $\mathrm{IPC}_{\mathrm{i}, \mathrm{t}}$ se refiere al indicador homónimo considerando su especificidad sectorial “i”, a través del tiempo " $t$ ”; mientras que para el caso de la TIIE diferenciación solamente considera su comportamiento a través de tiempo. A saber, la categorización sectorial se define por los siguientes rubros: Materiales, Industrial, Servicios y Bienes de Consumo Básico, Servicios y Bienes de Consumo Frecuente, Salud, Servicios Financieros, y Servicios de Telecomunicaciones.

Al respecto, tanto la tasa de interés, así como el Índice de Precios y Cotizaciones y sus respectivos sectores son de periodicidad mensual, tomando en cuenta el dato referente al cierre y su rango de observación comprende desde enero de 1998 hasta mayo de 2020.

Por su parte, debido a que se trabajará con series de tiempo, es requisito considerar la estacionariedad de cada una de las variables por medio de pruebas Dickey-Füller Aumentadas; sin embargo, dado que ninguna de las variables presenta un estadístico ADF menor a los niveles críticos de la prueba no es posible concluir que las series de datos en el nivel sean eficientes para proponer un modelo de estimación por medio de regresión y minimización de errores cuadrados (Hill, Griffiths y Lim, 2018). Por tal motivo se han 
obtenido las tasas de crecimiento interanuales de cada una de las series, a fin de definir por esta vía que presentan estacionariedad.

\section{RESULTADOS Y DISCUSIÓN}

En cuanto a los resultados de la metodología y modelos definidos en el apartado anterior, la tabla 1 muestra los resultados de estacionariedad referentes a las tasas de crecimiento de los IPC y la TIIE:

\section{Tabla 1.}

Prueba Dickey-Füller, variables en tasa de crecimiento.

\begin{tabular}{l|llll}
\hline Nombre & Estadístico ADF & Alfa 1\% & Alfa 5\% & Alfa 10\% \\
\hline TIIE** & -3.006 & -3.459 & -2.880 & -2.570 \\
IPC (General)** & -3.227 & -3.459 & -2.880 & -2.570 \\
IPC Sector Materiales** & -3.324 & -3.459 & -2.880 & -2.570 \\
IPC Sector Industrial** & -3.434 & -3.459 & -2.880 & -2.570 \\
IPC Sector Consumo no Básico** & -3.193 & -3.459 & -2.880 & -2.570 \\
IPC Sector Consumo & -3.641 & -3.459 & -2.880 & -2.570 \\
Frecuente*** & -2.932 & -3.459 & -2.880 & -2.570 \\
IPC Sector Salud** & -3.422 & -3.459 & -2.880 & -2.570 \\
IPC Sector Financiero** & & -3.459 & -2.880 & -2.570 \\
IPC Sector & -3.179 & & & \\
Telecomunicaciones** & & & & \\
\hline
\end{tabular}

Al respecto, la prueba de hipótesis en cuestión se refiere a $\mathrm{H} 0=$ no existencia de estacionariedad, mientras que la $\mathrm{H} 1=$ existe estacionariedad en la serie; de esta forma el rechazo de la hipótesis nula se define de la siguiente manera: *, rechazo al $10 \%$ de nivel de confianza de prueba alfa; **, rechazo al $5 \%$ de nivel de confianza de prueba alfa; ***, rechazo al $1 \%$ de nivel de confianza de prueba. Elaboración propia con base en Bolsa Mexicana de Valores (2020) e INEGI (2020).

Con base en los resultados de la tabla 1, se comprueba que las tasas de crecimiento de las variables sí son estacionarias; no obstante, al tratarse de variables proxy en diferencias se considera viabilidad para definir relación de bicausalidad de corto plazo por medio de un Modelo de Vectores Autorregresivos (VAR, por sus siglas en inglés) (Hill et al, 2018). 
Por todo lo anterior, se plantea el uso de un modelo VAR con la siguiente forma funcional:

$$
\left.\Delta \widehat{I P C}_{l, t}=b_{1,1} \Delta T I I E_{t}+b_{2,1} \Delta I P C_{i, t-1} \quad \text { (EC. } 3\right)
$$

Al respecto, la ecuación 2 se pueden representar la existencia de bicausalidad entre variables, así como el comportamiento cortoplacista originado por tasas de cambio de sus argumentos, lo cual entrega como resultado un test de impulso-respuesta. A saber, los modelos en cuestión son sustituidos en la variable IPC $_{\mathrm{i}, \mathrm{t}}$ para los modelos diferenciados por medio de la clasificación general y sectorial de la BMV con respecto al IPC.

De esta manera, se plantea un modelo de impulso respuesta para las combinaciones consideradas entre los índices bursátiles sectoriales y agregado del IPC y la Tasa de Interés Interbancaria de Equilibrio (TIIE), a las cuales se les ha anexado una diferencia de rezagos, considerando seis periodos mensuales.

Asimismo, la interpretación del modelo impulso respuesta se referirá al efecto que tiene la variable explicada del modelo de la ecuación 3 con respecto a la variable independiente de la misma ecuación; es importante considerar que se busca definir en qué rezago/eslabón/paso (el eje horizontal de todas las divisiones) se pierde el efecto de la variable impulso, así como la cuantía y dirección de dicho estímulo y contestación, como se observa tanto para el IPC general como en su desglose sectorial en la figura 4: 
Figura 4.

Impulso respuesta entre IPC General y sectorial
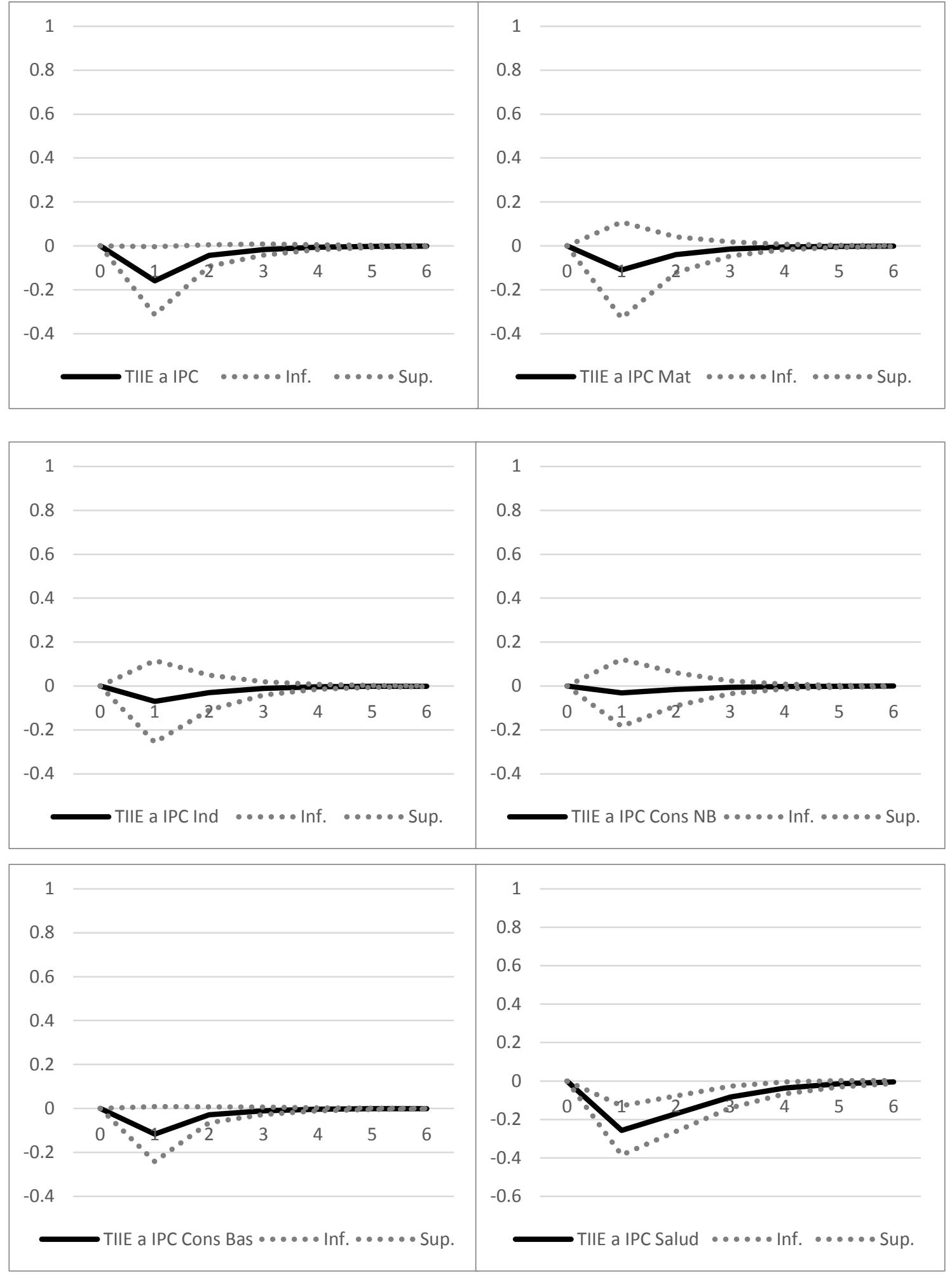

Aceves M., Torres A. D. y Hernández R. G

Análisis bursátil sectorial con relación a la tasa de interés en México 


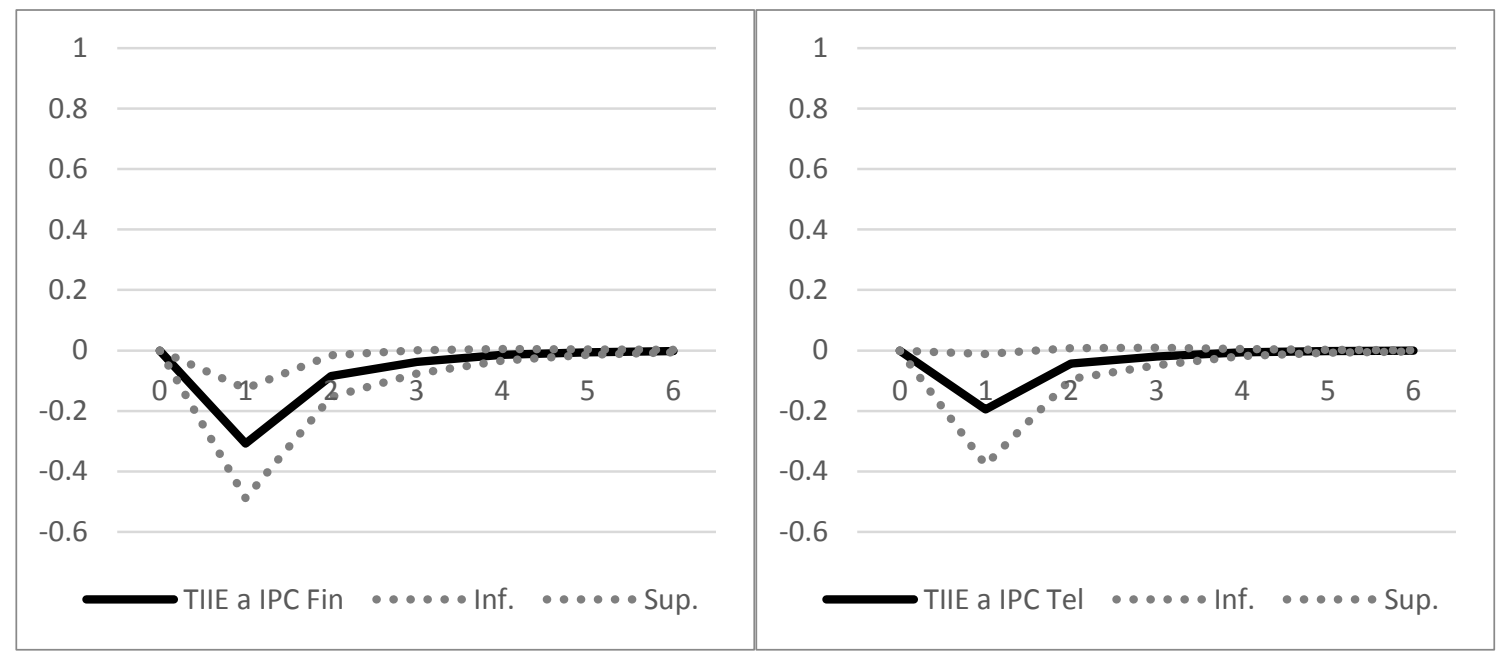

Elaboración propia con base en Bolsa Mexicana de Valores (2020) e INEGI (2020).

La figura 4 muestra la relación impulso respuesta entre la TIIE y el IPC tanto a nivel agregado como a nivel sectorial, mostrando el efecto sustitutivo y reductivo esperado. Particularmente, el primer elemento define la relación TIIE a IPC general, donde la aceleración en $1 \%$ de la tasa de interés, movimiento provocado por el uso de la Regla de Taylor, ocasiona una reducción del IPC en $-0.20 \%$ aproximadamente, efecto acumulativo que se pierde después de 5 meses. De esta manera, el impulso positivo de la TIIE produce una respuesta decreciente del IPC, la cual se mantiene hasta por 4 ó 5 meses.

Por su parte, los resultados en cuanto al análisis sectorial del IPC son heterogéneos; a saber, el impulso de la TIIE hacía el IPC Materiales es de una cuantía de $-0.1 \%$, es decir que el efecto restrictivo de la Política Monetaria de Objetivos de Inflación sobre las empresas productoras de materiales para construcción, químicos, metales y minería, plástico y papeleros es menor en comparación al promedio medido por el IPC. Por lo tanto, el sector Materiales es ciertamente insensible a la política monetaria de Banco de México.

De forma similar, el impulso de la TIIE sobre la tasa de crecimiento del IPC Industrial al igual que para para el IPC de Consumo No Básico entrega una respuesta bastante inelástica, con un valor menor al -0.1. Por lo tanto, el Sector Industrial y el para el Sector de Consumo No Básico en México, compuestos por ramas que abarcan al sector de infraestructura y servicio de transporte aeroespacial, maquinaria, construcción y equipo eléctrico pesado; así 
como comercialización y distribución de bienes duraderos, componentes automóviles, bienes de lujo, y hoteles, restaurantes y esparcimiento respectivamente, son insensibles a los mecanismos de Política Monetaria de la Regla de Taylor y por lo tanto se consideran sectores de inversión de menor volatilidad, o bien, dirigidos al beneficio y factibilidad de perfil inversionista conservador.

Si bien distintos sectores parecen presentar nula respuesta ante los impulsos restrictivos de la Regla de Taylor, los siguientes resultados muestran una relación de causalidad más significativa al Benchmark del mecanismo de la TIIE al IPC general. En el caso, el IPC Consumo Básico es de un valor de $-0.11 \%$, y que puede llegar incluso a ser de $-0.2 \%$, demostrando la significancia estadística del impulso de la TIIE sobre la respuesta del IPC que integra empresas del ramo bebidas, alimentos, productos domésticos y productos personales es de un $0.5 \%$ de confianza.

Por su parte, el impulso de la TIIE provoca una respuesta de -0.25 en el IPC Salud, la cual puede llegar hasta $-0.4 \%$. Por consiguiente, el Sector Salud es sumamente sensible a la aplicación de la Regla de Taylor, por lo que la inversión en empresas de biotecnología, equipo médico y medicamentos, productos farmacéuticos y servicios médicos, debe tomar en cuenta la dinámica de la inflación, dado que los incrementos de esta última por arriba de su objetivo de $3 \%$ tienen una importante afectación negativa sobre su crecimiento y rentabilidad.

Es así que la operación bursátil en empresas del sector salud, al observar el incumplimiento de la meta inflacionaria, deben cerrar posiciones para tomar beneficios y esperar hasta el periodo posterior a la ejecución de la Regla de Taylor donde los precios en el mercado bursátil ya habrían sido ajustados y se encontrarían en una fase de crecimiento.

En cuanto al IPC Servicios Financieros, el impulso de la TIIE produce la respuesta contractiva más significativa y amplia, con un valor de $-0.30 \%$, alcanzando un valor inferior de $-0.48 \%$. En específico, el IPC Servicios Financieros y por consiguiente las empresas del ramo bancos, bienes inmobiliarios, mercados financieros, seguros y 
SIEFORES, presentan la mayor sensibilidad con respecto a los movimientos de la TIIE; el mecanismo de transmisión es mayor en este sector debido a que la tasa de interés tiene una relación directa con respecto a la operación del mismo, al reducir el margen de beneficio directamente.

En otros términos, el incremento de la TIIE implica la reducción de ganancias en el sector financiero al incrementar el volumen de flujos de devolución que deben hacer las empresas hacía el Banco de México, y en el caso del mercado de valores, debido al efecto sustitución entre instrumentos de éste con respecto a aquellos que son de deuda.

Por lo tanto, el sector financiero es el de mayor sensibilidad con respecto a la tasa de interés, motivo por el cual los periodos de estabilidad inflacionaria serán pertinentes para realizar inversión en estos, pero ante escenarios de incumplimiento de meta de crecimiento de precios lo mejor es sustituir la inversión en este sector por aquellos que no presentan sensibilidad y, asimismo, volver a las emisoras del IPC Servicios Financieros ante el restablecimiento de la meta del Banco de México.

Finalmente, el análisis correspondiente al IPC Servicios de Telecomunicaciones se implica una respuesta de $-0.2 \%$ hasta $-0.39 \%$ ante impulsos positivos de la TIIE, es decir, las empresas dedicadas a los medios de comunicación y servicios de telecomunicación son ciertamente sensibles a la tasa de interés.

Por consiguiente, ante periodos de alta inflación, no es conveniente la inversión en el sector de telecomunicaciones, porque se tendrá como consecuencia el incremento de la tasa de interés y por lo tanto una reducción de beneficios.

Cabe señalar, que dicho margen de elasticidad refleja una mayor dependencia por el financiamiento mediante deuda, en comparación de la proporción de uso de recursos propios, por lo que el apalancamiento tiene una mayor importancia. Entonces, ante periodos de estabilidad en precios, las empresas de comunicación muestran una dinámica de beneficios destacable. 
De esta forma, con base en los resultados de la figura 4 es posible demostrar que las empresas que integran a los sectores de producción y distribución de materiales, industriales, y consumo no básico son insensibles a la política monetaria de Objetivos de Inflación; sin embargo, las emisoras pertenecientes a los sectores de consumo básico, salud, servicios financieros y telecomunicaciones son elásticas con relación a la ejecución de la Regla de Taylor $(1993,1998)$.

\section{CONCLUSIONES}

El presente trabajo de investigación consideró la realización de un estudio teórico-empírico sobre la relación perniciosa entre la tasa de interés con respecto a la dinámica del Índice de Precios y Cotizaciones de la Bolsa Mexicana de Valores, así como por la división sectorial determinada por la misma. Particularmente, se realizó una descripción y estudio de los hechos estilizados correspondientes a la inflación, tasa de interés, tipo de cambio, IPC y la división sectorial de este último, para finalmente considerar una serie de modelos de Vectores Autorregresivos capaces de identificar la cuantía predictiva entre estas variables. Es así como, los resultados obtenidos derivan en la estimación esperada, por lo cual la reproducción de la Regla de Taylor provoca reducciones en la dinámica bursátil, en términos generales. Por su parte, en cuanto a la división sectorial, los pertenecientes a materiales, industrial, y servicios de consumo no básicos presentaron un insignificante grado de sensibilidad ante los impulsos de la TIIE; sin embargo, para el caso de los sectores sobre servicios de consumo básicos, salud, servicios financieros y telecomunicaciones su grado de sensibilidad es bastante amplio, siendo superior a la propia elasticidad del IPC general.

De esta forma, las recomendaciones de inversión y seguimiento financiero recaen en el beneficio y factibilidad de consideración para la formación de portafolios adversos al riesgo, en emisoras que pertenecen a los sectores materiales, industrial, y servicios de consumo no básicos, dada la necesidad de cobertura ante las problemáticas de incertidumbre actuales, donde la recesión económica global requiere portafolios con mayor 
nivel de seguridad y reducción especulativa. No obstante, ante periodos de cumplimiento de la meta de inflación los sectores que sí son sensibles son más recomendables, pero se debe tener cuidado si se tienen posiciones abiertas en estos últimos y considerar el cierre de las mismas ante periodos de elevada inflación.

Es así como, la presente investigación aporta otros mecanismos que amplifican el análisis para la toma de decisiones en el ejercicio de inversión corporativa-bursátil, enriqueciendo las prácticas ya existentes en cuanto al uso del análisis técnico y fundamental.

\section{REFERENCIAS}

Banxico. (2020). Sistema de Información Económica (SIE). Recuperado de: https://www.banxico.org.mx/SieInternet/

Basistha, A., \& Startz, R. (2020). Monetary Shock Measurement and Stock Markets.

Bolsa Mexicana de Valores. (2020). Índices y Sectoriales Analíticos. Recuperado de: https://www.bmv.com.mx/es/indices/analiticos/

Figueroa, E., Pérez, F., y Godínez, L. (2016). El desempleo y la inflación en México. Opción, 32(13), 267-300.

García, A., y Perrotini, I. (2014). Modus operandi del nuevo consenso macroeconómico en Brasil, Chile y México. Problemas del desarrollo, 45(179), 35-63.

Granger, W. (1969). Investigation causal relation by econometric models and crossspectral methods.

Hallberg, M., \& Ryhage, M. (2019). Effects of Monetary Policy on Stock Market Liquidity: Empirical Analysis on the Swedish Market.

Hill, R. C., Griffiths, W. E., \& Lim, G. C. (2018). Principles of econometrics. John Wiley \& Sons.

INEGI. (2020). Banco de Información Económica (BIE). Recuperado de: https://www.inegi.org.mx/sistemas/bie/ 
Levine, R. (2004). FINANCE AND GROWTH: THEORY AND EVIDENCE. (10766). Cambridge, Massachusett, USA: NATIONAL BUREAU OF ECONOMIC RESEARCH. doi:10.3386/w10766.

Gómez, A. (2010). La relación entre el sistema financiero y el crecimiento económico en México. Proyecto de Investigación Aplicada. Monterrey, Nuevo León, México: Escuela de Graduados en Administación Pública y Politicas Públicas. Retrieved from https://repositorio.tec.mx/bitstream/handle/11285/629300/33068001090995.pdf?sequen ce $=1 \&$ isAllowed $=\mathrm{y}$

Montero, R. (2013). Test de Causalidad. España: Documentos en Economía Aplicada.

Pagano, M. (1993). Financial Markets and Growth. An Overview. European Economic Review. doi:10.1016/0014- 2921(93)90051-B

Taylor, J. B. (1993). Discretion versus Policy Rules in Praxtice. Carnegie-Rochester Conference Series on Public Policy, 39, 195-214. (S. University, Ed.) Standford, California, USA: North Holland. Retrieved from http://opendata.dspace.ceu.es/bitstream/10637/2345/1/p\%20195_214.pdf

Taylor, J. B. (1998). An Historical Analysis of Monetary Policy Rules. Stanford, California, USA: National Bureau of Economic Research. Retrieved from https://web.stanford.edu/ johntayl/Onlinepaperscombinedbyyear/1999/An_Historical_ Analysis_of_Monetary_Policy_Rules.pdf

Velázquez, D., \& Hernández, Z. (2013). Una revisión del papel del Sistema Financiero en el Crecimiento Economico (1ra Ed ed.). Hidalgo: Universidad Autonoma del Estado de Hidalgo.

Sánchez V., A. (2020). Estimación de la tasa de interés neutral, desempleo e inflación en México. Investigación económica, 79(311), 35-53.

Perrotini, I. (2014). El Nuevo consenso en teoría y política monetaria. En Rivas S. Aceves C., Castillo E. y Venegas F. (Editores), Teoría Económica: un panorama contemporáneo (pp. 1-34). Vol. I. México: Universidad Panamericana, Universidad de las Américas Puebla, IPN y Porrúa. 
Woodford, M. (2012). Inflation Targeting and Financial Stability. En Sveriges Riksbank Economic Review (pp. 7-32): NBER Summer Institute. 